\title{
Del escándalo del Tamiflu a una revolución de la evidencia científica en salud
}

\author{
Agustín Ciapponi* \\ Ciapponi A. Del escándalo del Tamiflu a una revolución de la evidencia científica en salud. Evid Act Práct Ambul. 2014, 17(2). Abr-Jun. 42-45.
}

\section{El resumen frío de los hechos}

Desde 2002 a la actualidad, los gobiernos de todo el mundo han gastado fortunas en inhibidores de la neuraminidasa (IN), tales como Tamiflu ${ }^{\circledast}$ (oseltamivir) de Roche y Relenza ${ }^{\circledast}$ (zanamivir) de GlaxoSmithKline (GSK), especialmente para enfrentar una eventual pandemia de Influenza.

Esta tendencia aumentó espectacularmente tras el brote del virus H1N1 (gripe porcina) en abril de 2009. Por entonces se consideraba que durante las pandemias de gripe los IN reducirían las complicaciones de la gripe como la neumonía, y las hospitalizaciones, lo que condujo a invertir sumas ingentes en la compra de medicación para proveer a la población. Sin embargo y como describiremos a lo largo de este artículo, la evidencia original presentada por los laboratorios a las agencias gubernamentales de todo el mundo para la aprobación de estos fármacos había sido incompleta y dudosamente verídica.

Neuraminidase inhibitors for preventing and treating influenza in healthy adults and children (Review)
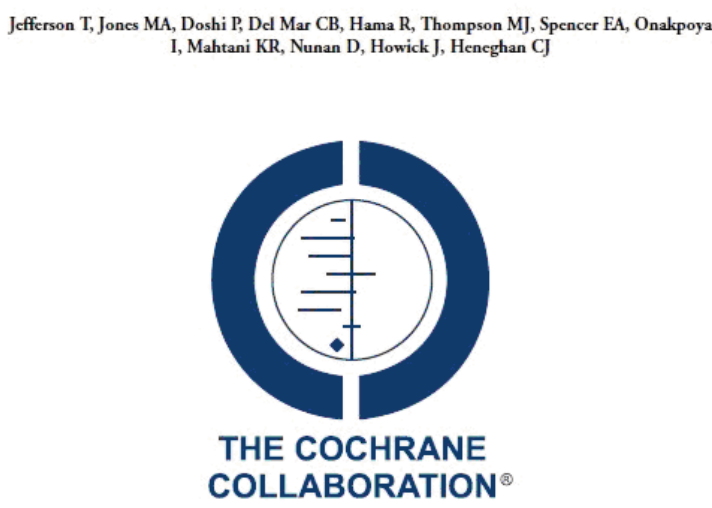

Un equipo internacional de investigadores con sede en Australia, Italia, Japón, Reino Unido y EE.UU., en colaboración con el Grupo Cochrane de Infecciones Respiratorias Agudas, extendió los límites de las revisiones sistemáticas típicas, que se basan fundamentalmente en el uso de los reportes de ensayos publicados, disponibles en revistas científicas.

En este caso, pudo constatarse que los reportes publicados en revistas cinéticas incluían una pequeña proporción de los datos existentes sobre la eficacia y la seguridad de los IN. Durante los últimos seis años, y tras la publicación de las primeras Revisiones Cochrane de oseltamivir y zanamivir, el Grupo Cochrane de Investigación de IN pudo acceder y revisar más de 160.000 páginas de documentos regulatorios con el fin de examinar el cuadro completo de la evidencia.

La última actualización de la Revisión Cochrane sobre IN, publicada el 10 de abril de 2014, se basa en los reportes internos completos de 20 ensayos de Tamiflu NR y 26 de Relenza'. Conducidos con anterioridad a la pandemia, habían incluido a más de 24.000 sujetos.
Documento avalado por la Asociación Argentina de Medicina Familiar y la Federación Argentina de Medicina Familiar y General

Los nuevos hallazgos, surgidos al evaluar toda esta información en conjunto, desafían el supuesto histórico de que los IN son realmente eficaces contra la influenza. La nueva revisión confirma pequeños beneficios en el alivio sintomático, como por ejemplo y en términos absolutos, acortar la duración promedio de los síntomas en sólo medio día (de 7 a 6,3 días) ${ }^{\S}$.

Por otro lado, la evidencia no sustenta que los IN reduzcan las internaciones o las neumonías confirmadas; y tampoco se encontró evidencia que sugiera que los IN prevengan la propagación de la gripe de persona a persona ya que al no hacerse cultivos virales de rutina, fue imposible descartar infecciones virales asintomáticas que igualmente pueden diseminar el virus.

Sin embargo, sí hay evidencia de los perjuicios a la salud vinculados con el uso de IN. Por ejemplo, los nuevos datos muestran que sólo necesitan tratarse 22 personas, para que ocurra un caso adicional de vómitos, 148 para un evento cardíaco, o 125 niños para desencadenar un evento adverso serio que obligue a suspender la medicación. Imaginemos la magnitud del daño si se extrapolara a millones de usuarios de IN. Frente a este nuevo cuadro de la evidencia, la relación riesgo/beneficio empeoró tan sustancialmente que resulta cuestionable mantener la misma política.

Los resultados encontrados por esta última revisión Cochrane publicada fueron posibles gracias al acceso a los reportes completos de investigación original (Clinical Study Reports, CSR), finalmente entregados por los fabricantes, Roche y GSK, tras una larga campaña de presión en ámbitos académicos, institucionales y en los medios de comunicación. Los resultados de la revisión cuestionan si el almacenamiento de IN por parte de los gobiernos sigue siendo justificable dada la falta de evidencia confiable que sustente los pretendidos beneficios.

\section{Las primeras repercusiones}

La publicación de la revisión Cochrane ha generado y sigue generando una gran atención en ámbitos académicos, en los medios de comunicación de todo el mundo y obviamente en las empresas farmacéuticas, principalmente por las implicancias a futuro y por el cambio de paradigma que está desencadenando.

Tom Jefferson y el resto de los autores de la revisión Cochrane expresaron que "...la aprobación de medicamentos y su uso ya no puede basarse más en información sesgada o ausente. Arriesgamos demasiado la salud de nuestra población y nuestra economía. Esta revisión Cochrane es la primera que se ha basado únicamente en reportes de estudios clínicos (CSR) y comentarios de agencias reguladoras. Es el primer ejemplo de lo que supone la ciencia abierta en la medicina, utilizando los informes de los estudios clínicos completos disponibles sin condiciones. Instamos a no confiar solo en los ensayos publicados o en comentarios de los expertos, con conflictos de interés, hasta no tener acceso a toda la información".

David Tovey, editor en jefe de la Biblioteca Cochrane, al comentar la publicación de la versión actualizada de la revisión sistemática Cochrane, dijo que "...esto demuestra la importancia de garantizar que los datos de los ensayos sean transparentes y accesibles"; mientras que Fiona Godlee, Editora en

* Servicio de Medicina Familiar y Comunitaria del Hospital Italiano de Buenos Aires. Centro Cochrane IECS. Instituto de Efectividad Clínica y Sanitaria. Ex Secretario de Asuntos Científicos de la Asociación Argentina de Medicina Familiar. Agustin.ciapponi@ hiba.org.ar

${ }^{\S}$ Vale aclarar que estos resultados surgen de comparar los IN contra placebo y en vez de hacerlo contra los medicamentos estándar para el alivio de los síntomas, como el paracetamol o los antinflamatorios no esteroideos. 


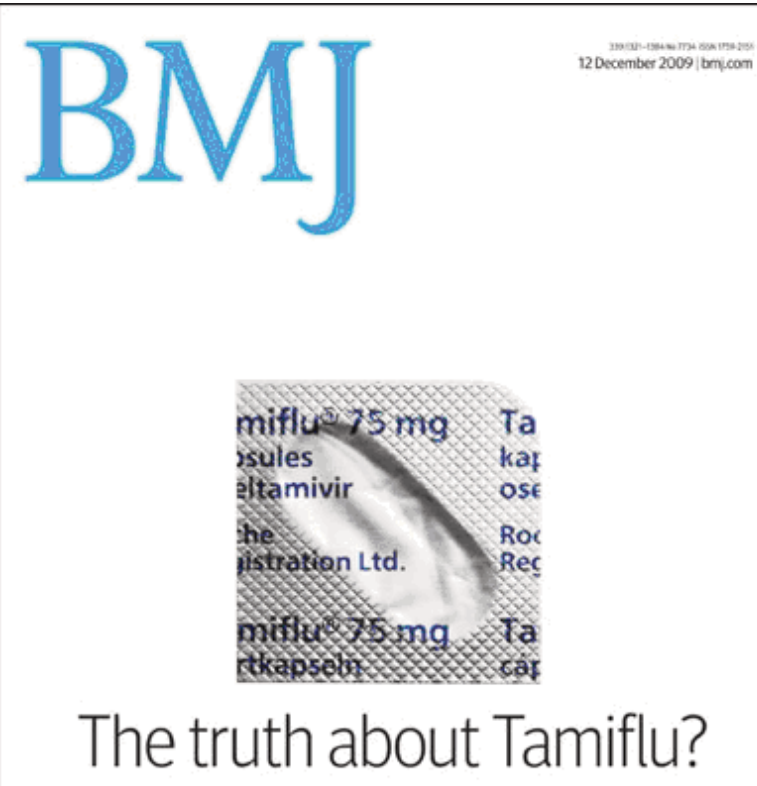

\author{
PLUS Hypothyroidism after pre-eclampsia \\ Managing hepatocellular carcinoma \\ Does weight loss improve sleep apnoea?
}

Jefe de la revista British Medical Journal, sostuvo que “...esta revisión es el resultado de muchos años de luchas para acceder y utilizar los datos de ensayos no publicados. Queda claro que las decisiones futuras para la compra y el uso de medicamentos, sobre todo a escala masiva, deben basarse en una visión completa de la evidencia, tanto la publicada como la no publicada. Necesitamos los datos completos de los ensayos clínicos de todos los medicamentos que se utilizan actualmente. La nueva Directiva Europea de Ensayos Clínicos nos señala el enorme desafío al que nos enfrentamos. Necesitamos el compromiso de las organizaciones y las compañías farmacéuticas para que todos los datos estén disponibles, incluso si eso significa que debemos remontarnos 20 años atrás. De lo contrario corremos el riesgo de otra reacción instintiva ante una posible pandemia. Y ¿realmente nos lo podemos permitir?"”.

Por otro lado, Ben Goldacre, autor del libro Bad Pharma ${ }^{3}$ y co-fundador de All-Trials", manifestó que "...el secreto que rodea a los ensayos clínicos significa que hay mucho que no sabemos acerca de otros medicamentos que tomamos...;...las compañías farmacéuticas ocultaron información por media década...;...la batalla del Tamiflu ilustra perfectamente la necesidad de una plena transparencia en torno a los ensayos clínicos, la importancia del acceso a la documentación oscura y el fracaso del sistema regulador"s; mientras que Elizabeth Loder y col. observaron que "...los estudios publicados incluyeron en algunos casos escritores fantasmas y a veces fue imposible determinar quién había llevado a cabo la investigación, lo que plantea serias dudas sobre rendición de cuentas y la independencia académica"6.

\section{Crónica de una batalla épica}

Las revisiones Cochrane de ensayos clínicos suelen ser el estándar de oro sobre la efectividad de las intervenciones y se actualizan permanentemente conforme se publican nuevos ensayos.
Además, permiten la inclusión de comentarios por parte de los lectores o revisores, lo que las transforma en evidencia viva.

La revisión Cochrane de IN publicada en 2008, había encontrado alguna evidencia de que los IN podrían reducir la tasa de complicaciones tales como la neumonía. Pero entonces un pediatra japonés llamado Keiji Hayashi dejó un comentario que activaría una revolución en nuestra comprensión de cómo debería funcionar la medicina basada en la evidencia. Esto no fue una publicación, ni siquiera una carta: era un simple comentario informal en Internet, publicado en el sitio en el sitio web Cochrane destinado a comentarios para cada revisión.

La revisión Cochrane había resumido los datos de todos los ensayos, explicó Hayashi, pero su conclusión positiva estaba fundamentalmente basada en los datos de sólo uno de los documentos: un resumen financiado por la industria de los diez ensayos existentes, que había sido publicado en 2003 en la revista Archives of Internal Medicine por Kaiser y col. ${ }^{7}$. De estos diez ensayos, sólo dos habían sido publicados en revistas revisadas por pares. Los ocho restantes, que habían incluído 4000 pacientes de todo el mundo, no habían sido publicados luego de diez años de haber finalizado y la única información disponible sobre sus métodos era un breve resumen de esta fuente secundaria.

Esta crítica fue considerada seriamente por Jefferson, autor principal de la revisión Cochrane 2008, quien junto a su equipo, se embarcó en la "aventura" de obtener la información necesaria hasta llegar a la verdad.

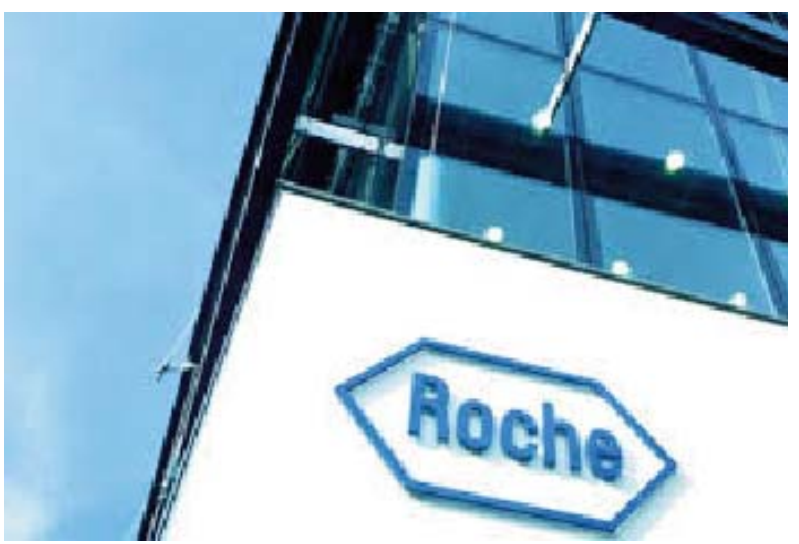

En primer lugar, los investigadores Cochrane escribieron al Dr. Kaiser que respondió que ya no tenía los archivos y que debían ponerse en contacto con Roche. Es aquí donde la aventura pareció transformarse en una misión imposible. Roche dijo que entregaría alguna información si los revisores Cochrane firmaban un acuerdo de confidencialidad. Esto resultaba complicado, ya que las revisiones Cochrane se cimentan en la transparencia absoluta de sus datos y su metodología, pero el contrato propuesto por Roche los obligaría a mantener la información en secreto y además mantener en secreto los términos del acuerdo. Roche estaba exigiendo entonces un contrato doblemente secreto, para que los investigadores pudieran acceder a datos sobre la seguridad y la eficacia de un medicamento que ha sido tomado por cientos de miles de personas en todo el mundo, para el que muchos gobiernos habían gastado sumas millonarias de millones. Esta inadmisible y lamentablemente muy usual demanda por parte de las autoridades del Laboratorio, no desanimó a Jefferson quién preguntó a Roche el por qué de la necesidad de firmar dicho contrato. La respuesta nunca llegó. 
En octubre de 2009, la empresa cambió de táctica. Afirmó que nada les gustaría más que entregar los datos, pero "casualmente" otra revisión académica sobre Tamiflu se estaba llevando a cabo, y dado que Roche le había dado a este otro grupo los informes de los estudios, Cochrane no podría tenerlos. Sin embargo, no existen razones por las que la misma cuestión no pueda ser estudiada por más de un grupo. De hecho, para temas tan polémicos y sensibles sería la opción preferible.

Una semana más tarde, Roche les envió a los autores de la revisión Cochrane siete extractos de los documentos internos de la empresa sobre los ensayos clínicos del meta-análisis de Kaiser. Fue un comienzo, aunque insuficiente para evaluar los beneficios, la seguridad o para entender cabalmente el diseño de los ensayos.

Al mismo tiempo, las inconsistencias en los datos parecían hacerse cada vez más ostensibles, ya que organizaciones de todo el mundo estaban arribando a conclusiones muy diferentes acerca de su efectividad. Por ejemplo, la Administración de Drogas y Alimentos (FDA) de EE.UU. no halló beneficios sobre complicaciones como la neumonía, mientras que el Centros para el Control y la Prevención de Enfermedades (CDC) de EE.UU. y la Agencia Europea de Medicamentos (EMA) sí encontraron beneficios.

Entonces, o estas organizaciones evaluaron datos diferentes o bien existía un desacuerdo razonable sobre la manera de interpretar la evidencia, en cuyo caso y para permitir un debate público transparente que informe a la comunidad médica académica y a los decisores de las naciones que compran el medicamento, sería imprescindible el acceso completo a los métodos y los resultados.

Es particularmente importante, en estos casos, determinar la validez metodológica de los estudios, ya que a menudo puede haber deficiencias en el diseño de un ensayo clínico, como fue el caso en muchos de los ensayos de oseltamivir (Tamiflu), que, por ejemplo, fueron "calificados" de "doble ciego" a pesar de que las cápsulas activas y las de placebo eran de diferentes colores. Pero lo más sorprendente fue que en casi todos los ensayos de oseltamivir, el diagnóstico de neumonía, probablemente el resultado clínico más relevante, fue definido por auto-reporte de los pacientes, cuando lo esperable sería un protocolo diagnóstico claro y reproducible, compuesto por al menos una radiografía de tórax y una evaluación ciega a la rama de tratamiento instituido, realizada por un médico clínico. De hecho, las complicaciones no constituían siquiera un resultado a evaluar en la mitad de los ensayos, o bien eran un resultado secundario o terciario en el resto.

Dado que se seguía negando la información necesaria para detectar estas fallas, los autores de la revisión Cochrane decidieron excluir todos estos datos de su análisis, dejando la revisión publicada en diciembre de 2009 a la espera de la información requerida. Si bien Roche publicó los extractos y se comprometió entonces a presentar los informes completos de los estudios disponibles, pasaron cuatro años sin que la empresa lo hiciera.

Esto no hizo más que ir incrementando las sospechas del mundo académico de que los extractos sobre los ensayos clínicos podrían estar incompletos y hasta ser engañosos. Era necesario mucho más detalle como el que proporcionan los CSR, documento intermedio entre los datos crudos y un artículo publicado en una revista. En éstos documentos se describe el plan de análisis, los roles cumplidos por cada investigador y descripciones detalladas de los eventos adversos.

Como ejemplo palmario de la inconsistencia y la insuficiencia de los datos disponibles, en los dos artículos publicados de los diez de la revisión de Kaiser, no se mencionaban eventos adversos graves pero sus CSR reportaban diez eventos adversos graves, tres de los cuales estuvieron posiblemente relacionadas con el Tamiflu. Por otro lado, uno de los ensayos más grandes nunca había sido publicado y rara vez se lo mencionaba en los documentos regulatorios.

A mediados del 2010, Roche comunicó que finalmente tenían lo requerido pero que le preocupaba la confidencialidad del paciente. Roche llevaba un año negando los reportes completos cuando viró su estrategia y empezó a plantear que algunos investigadores de la Colaboración Cochrane habían hecho declaraciones falsas sobre la droga y acerca de la empresa, pero se negó a decir quienes habían sido, qué habían dicho ni donde lo habían hecho. Expresó que "...difícilmente tuvieran la necesaria independencia para llevar adelante la revisión...". Aunque este cínico argumento hubiese sido cierto, ninguna empresa o investigador debería elegir quién tiene acceso a los datos de los ensayos, sino comprometerse con la verdad del conocimiento generado a costa de los pacientes participantes de dichos ensayos. Roche aún seguía negando los informes de los estudios.

Entonces Roche se quejó de que los revisores Cochrane habían empezado a "...copiar a los periodistas en sus mensajes de correo electrónico..." cuando respondían preguntas del personal de la empresa. Al mismo tiempo, comenzó a esgrimir que emitir juicio sobre los riesgos y beneficios y de las drogas es tarea de las agencias reguladoras y no de los académicos. Este argumento, inquietantemente familiar para cualquiera que haya seguido la campaña por mayor transparencia ensayos ${ }^{4}$, abría la siguiente pregunta: ¿es que las compañías se sienten más seguras con las agencias regulatorias que con la mirada crítica y transparente de la academia?

En primer lugar y al igual que con muchos otros medicamentos, los entes reguladores ni siquiera habían visto toda la información de los ensayos clínicos. Muchos, como la EMA, ni siquiera contaban con la capacidad de revisar la información internamente y debieron recurrir a evaluadores externos. Algunos de estos evaluadores casualmente habían aparecido en material promocional de Roche (se desconoce si los mismos lo habían declarado oportunamente a la EMA).

De esta forma, no debería sorprendernos que uno de cada cinco medicamentos tenga que retirarse del mercado en el plazo de cinco años por los graves efectos secundarios que provocan ${ }^{8}$. Dos notables ejemplos ocurridos en los últimos años fueron los de Vioxx ${ }^{\circledR}$ (rofecoxib) y Avandia $^{\circledR}$ (rosiglitazona). Éstos fueron comercializados y posteriormente retirados por los laboratorios Merck y GSK respectivamente debido al incremento del riesgo cardiovascular que ocasionaron a los pacientes que los recibieron. Estos efectos tampoco habían sido advertidos por las agencias reguladoras, sino por médicos y académicos independientes. Dejando de lado posibles sospechas de corrupción o de incompetencia, lo cierto es que la detección de sesgos en la determinación del balance entre los riesgos y los beneficios suele ser complejo, mientras que los estudios de farmacovigilancia son escasos e incompletos. Por esto es cada vez más necesario que múltiples observadores analicen libremente los datos. Por otro lado, los ensayos clínicos son cada vez mejor reportados (los estudios financiados por la industria cumplen con frecuencia las recomendaciones $\mathrm{CONSORT}^{9}$ ) y la presencia de problemas metodológicos ("trampas") puede ser verdaderamente sutil. Por ejemplo, en el caso de la rosiglitazona se mostraba mejoría estadísticamente significativa en un resultado compuesto de eficacia (cuestionable desde el punto de vista clínico), pero no así en los resultados individuales clínicamente importantes. O bien, otra trampa consistente en realizar comparaciones injustas. Por 
ejemplo los estudios que compararon los (costosísimos) antipsicóticos nuevos con dosis excesivamente altas de antipsicóticos tradicionales y, lógicamente documentaron una menor incidencia de efectos adversos en aquellos.

Finalmente, el año pasado, en un contexto favorable de cambio, después de media década de incesantes solicitudes de la Colaboración Cochrane y el British Medical Journal, Roche finalmente le dio al grupo Cochrane la información necesaria con la que finalmente se desmentirían los pretendidos beneficios que a su vez justificaban la compra masiva de oseltamivir por parte de muchísimos gobiernos.

Roche emitió un comunicado de prensa oponiéndose a estas conclusiones, pero sin dar razones. Ahora se podrá discutir, o no, a la revisión Cochrane, pero lo que es indiscutible es que todas las cartas ya están sobre la mesa y sólo sobre la base cierta de una revisión exhaustiva de la totalidad de la evidencia relevante podrá confirmarse o rebatirse la efectividad y seguridad de los IN, con argumentos estrictamente científicos.

Apoyamos la nueva normativa de ensayos clínicos aprobada por el Parlamento europeo por la cual las compañías farmacéuticas y los investigadores tendrán que publicar los resultados de todos los ensayos clínicos europeos en una base de datos accesible al público $^{10}$. Asimismo, resulta positivo el compromiso de la EMA en relación al acceso ${ }^{11}$ y a los cambios en los códigos de conducta, e incluso las promesas voluntarias de una mayor transparencia por parte de muchas empresas (Johnson \& Johnson, GSK, Roche ahora, y más) fruto de la campaña AllTrials ${ }^{4}$. Sin embargo, como se mencionó previamente, ya se han hecho promesas similares en el pasado que no logran concretarse salvo que exista un adecuado nivel de exposición y presión pública.

\section{Cambios de marea en la Agencia Europea de Medicamentos (EMA)}

El BMJ publicó recientemente una editorial titulada "Secretos en el cuidado de la salud"12 en contra de la participación en los debates públicos de grupos de presión al servicio de los intereses de las industrias, y denunciando la vuelta atrás de la EMA en relación con el acceso a los datos de los ensayos clínicos. Esta maniobra de la EMA para limitar la accesibilidad a los datos de los ensayos fue detallada por Ingrid Torjersen en otra publicación del $\mathrm{BMJ}^{13}$. Este proyecto de retroceso especifica que los informes de los ensayos, en lugar de ser publicados proactivamente por la agencia, estarían disponibles solamente para los usuarios registrados. Además, estos usuarios solo podrían acceder a la información únicamente como "vista de pantalla", sin que se les permita "descargar, guardar, editar, fotografíar, imprimir, distribuir o transferir la información" e imponiéndoles una estricta confidencialidad y amplias restricciones al uso de los datos.

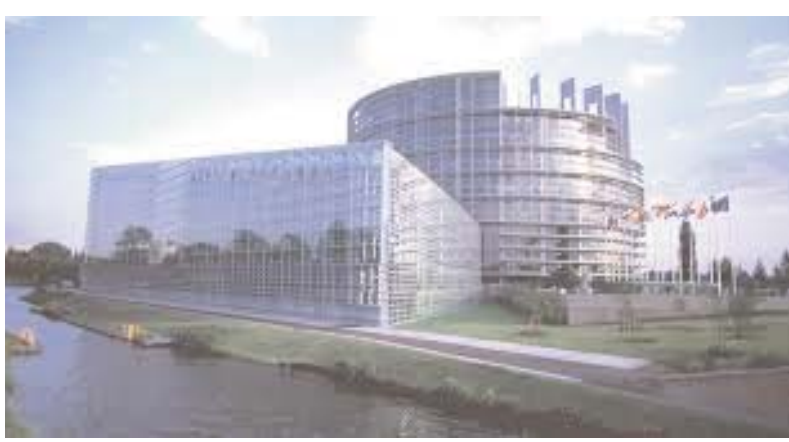

Otro documento relacionado reveló que la EMA se proponía no hacer accesible aquellos datos "...cuya divulgación puede poner en peligro la posición económica o de competencia del titular de la información".

Este cambio en la dirección de la Agencia se produjo después de una consulta a 169 empresas y organizaciones, 33 de las cuales eran compañías farmacéuticas o asociaciones de la industria.

Por otro lado, el año pasado, a dos compañías farmacéuticas estadounidenses, AbbVie y InterMune, se le concedieron las medidas cautelares que solicitaron a un tribunal europeo para prevenir que la EMA liberara documentos sobre la seguridad y la eficacia de sus fármacos. Después de que la EMA se mostrara de acuerdo con controlar las redacciones de los documentos que podían ser revelados, AbbVie retiró su demanda, justo un día después de que el Parlamento Europeo votara a favor de una iniciativa legislativa para hacer públicos los datos de los ensayos clínicos ${ }^{10}$.

Emily O'Reilly, la Defensora del Pueblo Europeo dijo: “...estoy preocupada por lo que parece ser un cambio significativo en la política de la EMA que podría minar el derecho fundamental del acceso del público a los documentos establecido por la ley de la UE..." y pidió a la EMA que defina "...cómo se propone hacer frente a las condiciones de acceso público establecidas en el Reglamento 1049/2001区

Tom Jefferson, primer autor de la revisión Cochrane dijo que “...cuando estábamos haciendo la revisión de Tamiflu tuvimos que cotejar la información todo el tiempo de atrás hacia adelante y de adelante hacia atrás. Los revisores, que se encontraban en todo el mundo y trabajaban a diferentes horas, necesitaban acceso inmediato. Si no se tiene acceso inmediato es como no tener acceso y, la impresión que yo tengo, es que ellos saben que es como no tener acceso. Creo que los revisores se han visto seriamente presionados por la industria".

Finalmente, se dio a conocer que la EMA revirtió esta última maniobra en respuesta a la presión de All trias y toda la comunidad académica. En la reunión de su junta ejecutiva del 12 de Junio, el Director Guido Rasi presentó enmiendas que eliminan la "vista de pantalla" como única posibilidad de acceso, permitiéndose la descarga de datos. Esta mejora sólo sucedió porque cientos de investigadores, los pacientes y los ciudadanos lo pidieron, ya que muchos de los asistentes a la junta no sabían de las críticas a la política de retroceso al acceso. La EMA está ultimando el texto de esta política que posiblemente se publicará en Septiembre.

Como es evidente, la participación activa ha cambiado las cosas y ha sido decisiva en muchas instancias de la guerra por la transparencia, que continuará hasta alcanzar el acceso a la información que todos necesitamos.

\section{Algunas lecciones del "Tamiflugate"}

El Tamiflugate se gestó cuando un equipo de obstinados académicos decidió oponerse a una cómoda y peligrosa inercia de basarse en un estudio publicado en una prestigiosa revista y luchar hasta que la compañía cediera la titánica pulseada. Tristemente, esta batalla tuvo lugar después de que los laboratorios se enriquecieran inmensamente con la compra masiva de IN por parte de los gobiernos. Pero esta resonante victoria no debe hacernos olvidar que los resultados completos de los ensayos clínicos sobre los medicamentos que utilizamos a diario están siendo aun rutinariamente y legalmente retenidos. No tomaremos decisiones informadas sobre qué tratamiento es el mejor mientras la información sobre los ensayos clínicos siga vedada a los tomadores de decisiones, profesionales de la salud, investigadores y pacientes. 
La buena noticia es que tras la batalla por el acceso a la evidencia del Tamiflu, algo ha cambiado para siempre, y si bien muchas cosas deberán seguir cambiando, los vientos por la búsqueda de la transparencia ya no podrán detenerse. Cualquier persona o grupo que se interponga en el camino de la transparencia expondrá a los pacientes a daños prevenibles. Necesitamos que reguladores, legisladores y cuerpos profesionales exijan transparencia total. Necesitamos conocer la información que falta, y quienes la retienen aun.

Por eso la campaña AllTrials", que ha tomado la bandera: "Todos los ensayos registrados, Todos los resultados reportados", ya cuenta con el apoyo de muchísimos organismos profesionales médicos y académicos en todo el mundo, así como más de cien grupos de pacientes e incluso de la compañía farmacéutica GSK. Cada vez se perciben más avances en cuanto a las mejoras en el acceso en la medida que el tema gana notoriedad en la sociedad. Este es un momento crucial en la historia de la medicina. La transparencia de los ensayos está finalmente en la gran agenda y existe una oportunidad única para lograrlo.

Es imperioso que a la vez que breguemos por los cambios normativos y legislativos necesarios alimentemos los cambios culturales, alentando y apoyando a las empresas y personas que están empezando a recorrer este camino. Debemos hacerles saber que es un desafío de toda la sociedad oponerse a aquellos que ponen los intereses comerciales de empresas por encima de la salud de la comunidad en su conjunto.

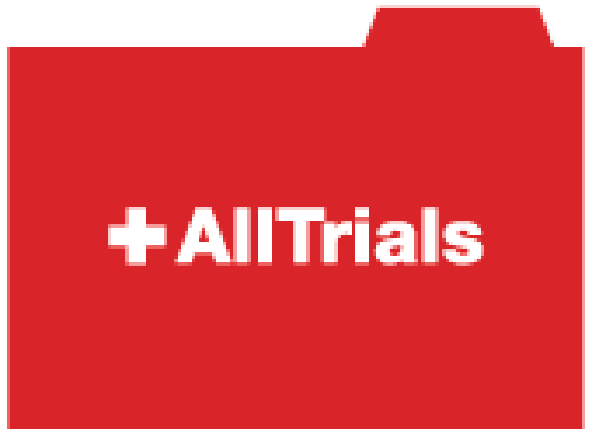

\section{Una mirada sobre la toma de decisiones}

¿Deberíamos haber gastado millones de este medicamento? Tras el resultado del brote de gripe porcina podríamos decir que no. Pero el análisis retrospectivo de las decisiones aunque parece sencillo, es intrínsecamente injusto (como se dice en el lenguaje futbolero, nadie se equivoca los resultados del domingo con el diario del lunes en la mano). El tema es definir si en esos días de abril de 2009, cuando no sabíamos si nos encontrábamos frente a una nueva pandemia de gripe se tomaron las mejores decisiones. Muy probablemente y dado que se carecía de la mejor información disponible en aquel momento histórico (estaba oculta para los tomadores de decisiones), es muy probable que la decisión tomada no haya sido la más conveniente para las naciones que invirtieron importantes sumas de sus presupuestos en la compra de dichos antivirales sin poder sopesar los riesgos y los beneficios reales del uso de los IN.

Aunque las autoridades podrían no haberlo interpretado así, nunca documentaron inequívocamente las razones para aprobar el almacenamiento de los IN. En este contexto, la decisión parece haberse basado en el supuesto de que los fármacos reducirían a la mitad los ingresos hospitalarios y las complicaciones graves de la gripe, y que frenarían la propagación viral ${ }^{14,15,16}$.

Aunque estas asunciones se basaban fundamentalmente en el estudio de Kaiser y col. ${ }^{7}$ que hasta entonces parecía una evidencia científica de alta calidad, la mayor parte de los datos incluidos en ese meta-análisis financiado por la industria, provenían de fuentes no publicadas e inaccesibles a la evaluación independiente. Además, las inconsistencias entre la FDA, que no halló evidencias de los beneficios son sobre complicaciones, y la EMA y el CDC, que sí lo hicieron, debieron haber encendido aún más la luz de alarma de los decisores.

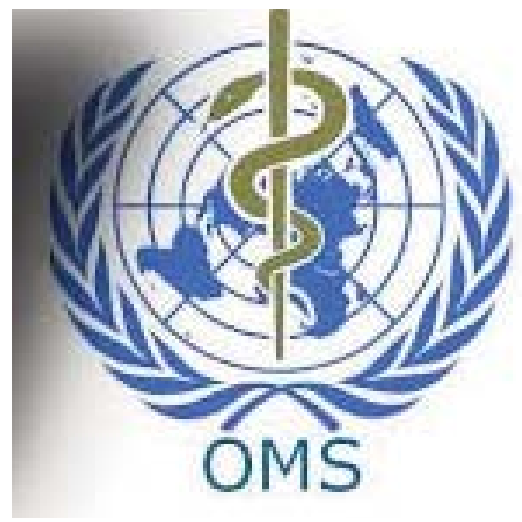

Organismos de salud pública, como el CDC y la Organización Mundial de la Salud (OMS) deberían considerar la nueva evidencia y revisar sus recomendaciones. Sin embargo el CDC respalda a los IN ahora basándose en estudios observacionales retrospectivos, muchos de los cuales tienen gran cantidad de datos faltantes y carecen de ajuste por el sesgo de sobreviviente (donde los que mueren antes tienen menos oportunidades de recibir tratamiento) ${ }^{17}$. Del mismo modo, la OMS todavía considera al oseltamivir suficientemente importante como para colocarlo en la lista de medicamentos esenciales que deben estar universalmente disponibles ${ }^{18}$, basándose en las mismas asunciones no probadas que apoyaron los planes ante la pandemia.

Esto no debería sorprendernos si tenemos en cuenta que las revisiones sistemáticas rara vez se utilizan para la elaboración de recomendaciones. En cambio, los procesos suelen depender en gran medida de la opinión de expertos en una especialidad concreta, en lugar de la de los representantes de la población a la que se aplican las recomendaciones, o de la de metodólogos expertos. $^{19}$

Vale destacar que los responsables de las agencias de regulación reportaron haber estado bajo presión política para proporcionar una solución farmacéutica a la amenaza de una pandemia ${ }^{20}$. Por su parte, la OMS informó haber sido influenciada por líderes de opinión pagados por la industria ${ }^{21}$. Ante tales cuestionamientos a la independencia de criterio, surge la pregunta de si el sistema regulatorio actual de organizaciones nacionales y globales es adecuado para garantizar una toma racional de las medidas destinadas a la protección de la salud.

Con estos hechos ha quedado demostrado con mayor detalle que nunca antes, que la totalidad del ecosistema de instituciones y procedimientos concernientes a la evaluación técnica, aprobación y regulación de medicamentos está bajo serio cuestionamiento. Aún quedan importantes batallas por librar antes de que tengamos un sistema de evaluación y regulación de drogas realmente al servicio de los pacientes y del interés público. Tenemos que actuar para asegurarnos de que las decisiones futuras no se basen en datos incompletos; menos aun cuando se trata de decisiones de impacto global, con altos costos potenciales y en un contexto de información controversial. 


\section{La evidencia revolucionada}

¿Deberíamos confiar en las publicaciones revisadas por pares? Está claro que este saludable proceso, aunque necesario, es insuficiente. Las revistas deberían exigir el acceso libre a los CSR, tal como lo han hecho con el registro de ensayos, de manera que los revisores de pares y cualquier lector pueda consultarlos. De esta forma se alinearían al objetivo de acceso libre e irrestricto al material educativo, como el propiciado por el movimiento "open access" 2 .

Por otro lado, en cuanto a la síntesis de evidencia, ni los tiempos límite, ni las actuales estructuras de financiamiento proveen los recursos suficientes como para analizar los extensos y detallados CSR. A diferencia de la información acerca de un ensayo contenida en un artículo de una revista que suele tener una extensión de menos de diez páginas, un CSR puede tener cientos o miles. Esto implica que la información tenga que ser terriblemente resumida o "comprimida"** (como suele decirse en la jerga editorial).

Ejemplos como el del "Tamiflugate" ponen de manifiesto que los financiadores deberían considerar una asignación de recursos acorde a la importancia de la problemática que estén investigando ya que de cambiar las políticas a favor de la transparencia los CSR, se harán públicos la mayoría de sus procesos con más frecuencia. Al mismo tiempo, las metodologías de síntesis deberán hacerse más rápidas y eficientes para asistir a los decisores de las instituciones reguladoras y a los políticos, médicos clínicos y pacientes en la tarea de proporcionar evidencia de alta calidad y, fundamentalmente, en forma oportuna. Asimismo, deberían revi- sarse los métodos utilizados para la toma de decisiones de cobertura, incorporando las revisiones sistemáticas, que proporcionan el mayor nivel de evidencia científica.

La Colaboración Cochrane, que está liderando esta iniciativa, lo manifiesta con claridad en su estrategia 2020 y ha lanzado fuertes incentivos financieros a los grupos de investigadores para "cambiar el juego" y dar cuenta metodológica y tecnológicamente de este desafío .

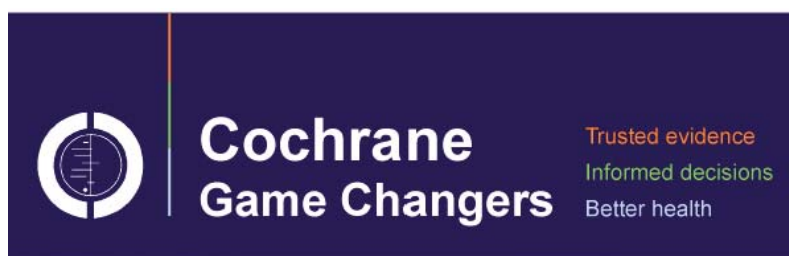

Los autores de la revisión Cochrane de IN se vieron obligados a convertirse en pioneros, adaptando la metodología de revisión sistemática (como la herramienta de evaluación de calidad) y desarrollando nuevas alianzas frente a la magnitud de la tarea. Así, aun sin proponérselo, han establecido nuevos estándares para los revisores sistemáticos y para los tomadores de decisión. Este quizás sea el primer ejemplo de lo que supone la ciencia abierta en la salud, alcanzado gracias a esfuerzos encomiables. Por eso mismo, es nuestra obligación abrazarlo y difundirlo para que se multiplique hasta que sea literalmente irrefrenable.

El autor agradece a los Dres Ariel Bardach y Demián Glujovsky por su exhaustiva revisión y significativos aportes a este artículo editorial.

\section{Referencias}

1. Jefferson T, Jones MA, Doshi P, Del Mar CB, Hama R, Thompson MJ, et al. Neuraminidase inhibitors for preventing and treating influenza in healthy adults and children. Cochrane Database Syst Rev 2014;4:CD008965. Disponible en: http://onlinelibrary. wiley.com/doi/10.1002/14651858.CD008965.pub4/pd

2. Godlee F. Clinical trial data for all drugs in current use. BMJ. 2012-10-29 16:10:15 2012;345.

Bad Pharma: How drug companies mislead doctors and harm patients, Fourth Estate, 2012 (UK). ISBN 978-0-00-735074-2

4. All trials. All Trials Registered | All Results Reported. Disponible en http://www.alltrials.net/
5. What the Tamillu saga tells us about drug trials and big pharma por Ben Goldacre, The Guardian , 10 de abril 2014, Disponible en: http://www.theguardian.com/business/2014/apr/10/tamiflu-saga-drug-trialsbig-pharma

6. Loder E, Tovey D, Godlee F. The Tamiflu trials. BMJ. 2014;348:g2630.

7. Kaiser L, Wat C, Mills T, Mahoney P, Ward P. Hayden F. Impact of oseltamivir treatment on influenza-related lower respiratory tract complications and hospitalizations. Arch Intern Med 2003:63:1667-72. 8. Entrevista con el especialista Víctor Montori: Uno de cada cinco fármacos se retira al plazo de cinco años por sus efectos adversos. http://www.elcorreo.com/alava/v/20120614/sociedad/cada-cinco-farmacos-

9. Moher D, Hopewell S, Schulz KF, et al. CONSORT 2010 explanation and elaboration: updated guidelines for reporting parallel group randomised trials. BMJ. 2010;340:c869.

10. Ensayos clínicos: reglas claras y una mejor protección para los pacientes. Disponible en: http://www.nogracias.eu/2014/04/03/ensayos-clinicos-reglas-claras-y-una-mejor-proteccion-para-los-pacientes/ 11. Eichler HG, Abadie E, Breckenridge A, Leufkens H, Rasi G. Open clinical trial data for all? A view from regulators. PLoS medicine. 2012;9(4): 100101202.

11. Eichler HG, Abadie E, Breckenridge A, Leufkens H, Rasi G. Open clinicalt
12. Ladher N. Secrets in healthcare. BMJ. 2014-05-22 11:26:48 2014;348.

12. Ladher N. Secrets in healthcare. BMJ. 2014-05-22 11:26:48 2014;348.
13. Torjesen I. European drug agency backtracks on plan to give researchers access to clinical trial reports. BMJ. 2014-05-21 06:30:59 2014;348.

14. UK Department of Health. UK influenza pandemic contingency plan. 2005. Disponible en: www.dh.gov.uk/prod_consum_dh/groups/dh_digitalassets/@ dh/@en/documents/digitalasset/dh_4121744.Pdf

15. US Department of Health and Human Services. HHS Pandemic Influenza Plan. 2005. Disponible en: www.pandemicflu.gov/planning-preparedness/federal/hhspandemicinfluenzaplan.pdf.

16. World Health Organization. WHO interim protocol: rapid operations to contain the initial

emergence of pandemic influenza. Disponible en: www.who.int/entity/csr/disease/avian influenza/guidelines/RapidContProtOct15.pdf.

17. US Centers for Disease Control and Prevention. CDC recommendations for influenza

antiviral medications remain unchanged. CDC Newsroom, 7 February 2012. Disponible en: http://www.cdc.gov/media/haveyouheard/stories/lnfluenza_antiviral.html

18. WHO Model Lists of Essential Medicines. Acceso el 12/6/2014 en: http://www.who.int/medicines/publications/essentialmedicines/en/

19. Oxman AD, Lavis JN, Fretheim A. Use of evidence in WHO recommendations. Lancet. Jun 2 2007;369(9576):1883-1889.

20. Cohen D. Complications: tracking down the data on oseltamivir. BMJ 2009;339:b5387.

21. Cohen D, Carter P. WHO and the pandemic flu "conspiracies." BMJ 2010;340:c2912.

22. Iniciativa de Budapest para el Acceso Abierto. Disponible en: http://www.budapestopenaccessinitiative.org/translations/spanish-translation

23. Cochrane Strategy to 2020. Disponible en: http://www.cochrane.org/community/organisation-administration/cochrane-strategy-2020 